\title{
EVALUACIÓN DE LA MOVILIDAD DEL SEMEN CRIOPRESERVADO DE CABALLOS CRIOLLO COLOMBIANO POR UN SISTEMA ANALIZADOR DE CLASE
}

\author{
ASSESSMENT OF CRYOPRESERVED SPERM MOTILITY \\ FROM COLOMBIAN CREOLE STALLIONS BY SPERM CLASS \\ ANALYZER
}

\author{
Giovanni Restrepo ${ }^{1}$, Daniel Ocampo ${ }^{2}$, Andrés Velásquez ${ }^{3}$
}

\begin{abstract}
${ }^{1}$ Médico Veterinario, cPhD. Facultad de Ciencias Agrarias. Politécnico Colombiano Jaime Isaza Cadavid. Carrera 48 No 7-151 Of. P19-117. Medellín, Colombia. E-mail: grestrepo@elpoli.edu.co; ${ }^{2}$ Ingeniero Agropecuario. Facultad de Ciencias Agrarias. Politécnico Colombiano Jaime Isaza Cadavid. Transversal 27 A sur $N^{\circ}$ 42-46. Antioquia, Colombia. E-mail: danielocampo86261@elpoli.edu.co; ${ }^{3}$ Ingeniero Agropecuario. Facultad de Ciencias Agrarias. Politécnico Colombiano Jaime Isaza Cadavid. Carrera 54 A Nº 75 Sur 10. Antioquia, Colombia. E-mail: andres_velasquez86103@elpoli.edu.co
\end{abstract}

Rev. U.D.CA Act. \& Div. Cient. 16(2): 445-450, Julio-Diciembre, 2013

\section{RESUMEN}

La reproducción juega un papel fundamental en el mejoramiento genético de los equinos. La criopreservación del semen equino es una técnica complementaria a los procesos de biotecnología reproductiva; sin embargo, solamente el $40 \%$ de los caballos producen semen que se puede criopreservar, con una alta variabilidad en su fertilidad. La confiabilidad de los métodos de evaluación del semen es trascendente para determinar su fertilidad potencial; no obstante, el análisis convencional por evaluación microscópica ha sido poco efectivo, en este sentido. El análisis de semen asistido por computador (CASA) permite una medición objetiva de la movilidad de semen. Esta investigación tuvo como objetivo comparar la evaluación de la movilidad del semen equino, mediante los métodos convencional y SCA ${ }^{\circledR}$ (CASA). El semen de tres caballos de la raza criollo colombiano (tres eyaculados cada uno) fue congelado, en un diluyente ( $4 \%$ de yema de huevo, $5 \%$ de dimetilformamida). La movilidad del semen descongelado, se determinó de forma convencional por microscopia de contraste de fase y mediante el software $\mathrm{SCA}^{\circledR}$. Para el análisis estadístico, se ajustó un modelo lineal generalizado (GLM) y se determinó la asociación entre las variables, a través de análisis de correlación y regresión. Se hallaron promedios de movilidad total de $54,3 \pm 10,1$ y 61,8 $\pm 13,9$, para evaluación convencional y por el sistema SCA ${ }^{\circledR}$, respectivamente. No se encontró diferencia significativa entre los métodos de análisis. Se concluye que el análisis de semen equino, mediante el método convencional y el sistema
$\mathrm{SCA}^{\circledast}$, es equiparable, para analizar la movilidad del semen equino criopreservado.

Palabras clave: Biotecnología, fertilidad, movilidad espermática, CASA.

\section{SUMMARY}

Reproduction plays a key role in the genetic improvement of horses. Equine semen cryopreservation is a complementary technique to reproductive biotechnology processes. However, only $40 \%$ of the horses produce semen with a high variability in fertility that can be cryopreserved. The reliability of methods of semen evaluation is important to determine their fertility potential, however the conventional analysis by microscopic evaluation has not been very effective in this regard. Computer-assisted semen analysis (CASA) provides an objective measurement of sperm motility. This study aimed to compare the evaluation of equine sperm motility through conventional and $\mathrm{SCA}^{\oplus}$ (CASA) methods. Semen of three horses of the Colombian creole breed (three ejaculates each) was frozen in extender (4\% egg yolk, $5 \%$ dimethylformamide). Thawed sperm motility was conventionally determined by phase contrast microscopy and by SCA $®$ software. For statistical analysis a generalized linear model (GLM) was fitted and the association between variables, using correlation and regression analysis, was determined. An average of total motility of $54.3 \% \pm 10.1 \%$ and $61.8 \% \pm 13.9 \%$ for conventional and $\mathrm{SCA}^{\circledast}$ evaluation, 
respectively, was detected.. No significant difference was observed between the methods of analysis. It is concluded that equine semen analysis by conventional method and $\mathrm{SCA}^{\circledR}$ system is comparable for analyzing the motility of criopreserved equine semen.

Key words: Biotechnology, fertility, sperm motility, CASA.

\section{INTRODUCCIÓN}

En Colombia, la especie equina es cada día más importante, debido a su amplia participación en el trabajo, en actividades deportivas y de exposición, de acuerdo a los diferentes andares. La reproducción juega un papel fundamental en el mejoramiento genético de dicha especie, por lo cual, la posibilidad de disponer de material seminal de excelente calidad para procedimientos, como la inseminación artificial, permite alcanzar altos estándares de eficiencia reproductiva.

La criopreservación es una técnica de gran importancia para la industria equina, ya que favorece el transporte y el almacenamiento de semen a largo plazo (Loomis \& Graham, 2008). Tradicionalmente, la evaluación del semen se ha basado en el trabajo manual de personas que, debido a su capacitación y su experiencia, han obtenido resultados prácticos y satisfactorios (Stephens et al. 1988). Con el fin de predecir la fertilidad del semen equino, el análisis de rutina es basado en la evaluación macroscópica-física del eyaculado y en la evaluación por microscopía de los espermatozoides (Pesch et al. 2006); sin embargo, el análisis convencional ha sido poco efectivo en la predicción de la fertilidad del semen (Kuisma et al. 2006).

El análisis de semen asistido por computador (CASA) ha permitido una medición objetiva de muchos parámetros de la movilidad y la morfología de semen, ofreciendo mediciones más confiables, imparciales y repetibles, respecto al examen visual (Magistrini et al. 1996; Colenbrander et al. 2003; Graham \& Mocé, 2005). Mediante sistemas CASA, se ha encontrado una mayor asociación entre la movilidad y la fertilidad, medida a través de parámetros, como la tasa de preñez estacional, el porcentaje de preñez por ciclo y el porcentaje de preñez al primer ciclo (Love, 2011).

La aplicación práctica del sistema CASA, como parte del mejoramiento en la calidad de los análisis del semen del caballo, se ha visto limitada por aspectos, como la carencia de valores estándar definidos, para lo que es normal y anormal en el movimiento del espermatozoide, al uso de referentes seleccionados sin criterios estadísticos y por la falta de implementación de una reglamentación internacional, para los ajustes del equipo (Katila, 2001; Quintero et al. 2003; Hoogewijs et al. 2011).

Con el avance tecnológico, se han mejorado los sistemas
CASA; un sistema reciente es el SCA ${ }^{\circledR}$ (Sperm Class Analyzer; Microptic S.L.), comparado en la evaluación de semen humano, con el sistema IVOS ${ }^{\circledR}$ y con la evaluación convencional manual no computarizada, encontrando resultados equiparables (Proctor et al. 2009). El sistema SCA ${ }^{\circledR}$ ha sido previamente empleado para la evaluación de semen equino (Hidalgo et al. 2005; Mesa \& Henao, 2012); sin embargo, es necesario establecer la relación entre sus resultados y aquellos hallados de forma convencional.

Esta investigación tuvo como objetivo evaluar la movilidad del semen de caballos de la raza criollo colombiano, mediante un sistema $\mathrm{SCA}^{\circledR}$ y compararlo con la evaluación microscópica convencional.

\section{MATERIALES Y MÉTODOS}

Recolección de semen. El estudio, se desarrolló con tres ejemplares equinos de raza criollo colombiano, ubicados en el municipio de Girardota (Antioquia), sometidos a condiciones iguales de alimentación, ambiente, manejo general y reproductivo. Con una condición corporal de 6 a 7 (1-9). El procedimiento de recolección, se realizó utilizando una yegua y una vagina artificial modelo Missouri (Minitube), lubricada con gel no espermicida. La fracción en gel de cada eyaculado fue removida por filtración. Se recolectaron tres eyaculados por animal, con un periodo de descanso sexual máximo de ocho días.

Evaluación del semen fresco. A cada eyaculado recolectado, se le determinó la movilidad total, al promediar los porcentajes de movilidad de cinco diferentes campos de observación (400X), en un microscopio de contraste de fase (Nikon E200) y se determinó la concentración espermática por espectrofotometría (Spermacue ${ }^{\circledR}$, Minitube) (Jasco, 1992). Como criterios de selección, solamente se procesaron los eyaculados, con un mínimo de $60 \%$ de movilidad y una concentración mínima de 100 millones de espermatozoides por mL.

El semen recién recolectado fue mantenido a $37^{\circ} \mathrm{C}$ y diluido en proporción $1: 1$, en un diluyente a base de leche descremada precalentado y antibióticos (EquiPro ${ }^{\circledR}$, Minitube), a la misma temperatura; luego, el semen fue transportado en refrigeración a $5^{\circ} \mathrm{C}$, al laboratorio de Biotecnología Animal.

Criopreservación del material seminal. La criopreservación del semen, se realizó por congelación rápida en vapores de nitrógeno, mediante un protocolo modificado del reportado por Bustamante et al. (2009). Para lo cual, el eyaculado fue centrifugado a 3000rpm por 15 minutos; luego, el precipitado fue suspendido en $2 \mathrm{~mL}$ de diluyente de congelación, compuesto por diluyente base (EquiPro ${ }^{\circledR}$ ) suplementado con $4 \%$ de yema de huevo y $5 \%$ de dimetilformamida (DMF). Posteriormente, el semen fue resuspendido en este mismo 
medio, en cantidad suficiente, para una concentración final de 100 millones de espermatozoides/mL

El semen diluido se mantuvo en refrigeración a $4^{\circ} \mathrm{C}$ por 15 minutos y se empacó en pajillas de $0,5 \mathrm{~mL}$. Las pajillas fueron nuevamente refrigeradas a $4^{\circ} \mathrm{C}$ por 10 minutos y se sometieron a vapores de nitrógeno líquido, durante 15 minutos, al ser puestas horizontalmente a una distancia de $4 \mathrm{~cm}$ de la superficie de este. Finalmente, se sumergieron en un tanque de almacenamiento de nitrógeno líquido a $-196^{\circ} \mathrm{C}$.

Evaluación del semen post descongelación. Después de mínimo una semana de almacenamiento del semen, se realizó la descongelación de pajillas, a $37^{\circ} \mathrm{C}$ por 60 segundos. La determinación de la movilidad espermática post-descongelación y sus parámetros, se realizó mediante dos métodos, desarrollados simultáneamente por los mismos técnicos evaluadores sobre cada pajilla descongelada. El primer método correspondió a la evaluación convencional, a través de microscopia de contraste de fase en cinco campos visuales diferentes (400X) y, el segundo, por medio de un sistema de análisis computarizado $\mathrm{SCA}^{\circledR}$.

Para el análisis, mediante el sistema $\mathrm{SCA}^{\circledR}$, se utilizó un procedimiento modificado del reportado por Hidalgo et al. (2005), para lo cual, se empleó un microscopio de contraste de fase (Nikon E200), con una cámara digital (Basler Scout SCA780), adaptada a un computador dotado del software SCA ${ }^{\circledR}$ Motility and Concentration (Microptic S.L.). Se estableció una configuración específica para el software de: cámara de cubreobjetos de $20 \mathrm{~mm}$ por $20 \mathrm{~mm}$, óptica en lente de ph-, gota de $8 \mu \mathrm{L}$ especie caballo, platina térmica a $37^{\circ} \mathrm{C}$ y un tamaño de partícula de 20 a $72 \mu \mathrm{m}$. La dilución del semen que se usó para el análisis fue de 1:0,5, empleando $500 \mu \mathrm{L}$ de semen en $250 \mu \mathrm{L}$ de citrato de sodio temperado a $37^{\circ} \mathrm{C}$.

Se evaluaron los porcentajes de movilidad total y de movilidad progresiva, que se definieron en función de la velocidad curvilínea (VCL) de los espermatozoides y, a su vez, fueron clasificados, según diferentes patrones de movilidad (Miró et al. 2005). De igual forma, se analizaron los parámetros: velocidad curvilínea (VCL), velocidad rectilínea (VSL), velocidad media (VAP), índice de linealidad (LIN), índice de rectitud (STR), índice de oscilación (WOB) y los parámetros de angularidad y oscilación de la cabeza: amplitud del desplazamiento lateral de la cabeza (ALH) y frecuencia de batida (BCF) (Mortimer, 1997; Rodríguez et al. 2011).

Análisis estadístico. Se realizó un diseño completamente al azar y los datos fueron analizados, mediante un modelo lineal generalizado (GLM), para la variable dependiente movilidad total del semen descongelado. Las medias para cada método de evaluación (convencional y SCA ${ }^{\circledR}$ ), se compararon por la prueba de Tukey.
El modelo empleado fue: $\mathrm{Y}_{i j k l m}=\mu+\mathrm{E}_{i}+\mathrm{M}_{j}+\mathrm{R}_{k}+\mathrm{RM}_{l}$ $+\mathrm{e}_{i j k l m}$, donde:

\section{$\mathrm{Y}_{i j k l m}: \quad$ Movilidad total}

$\mu$ : $\quad$ Media común a todos los tratamientos

$\mathrm{E}_{i}: \quad$ Efecto fijo del equino $(i) .(i=1 \ldots 3)$

$M_{j}$ : Efecto fijo del método de evaluación (j). $\left(j=\right.$ Convencional, SCA ${ }^{\circledR}$

$\mathrm{R}_{k}: \quad$ Efecto fijo de la pajilla $(k) .(k=1 \ldots 10)$

$\mathrm{RM}_{l}$ : $\quad$ Efecto fijo de la interacción de la pajilla por el método $(l=0 \ldots 20)$

$\mathrm{e}_{\mathrm{ijklm}}: \quad$ Error aleatorio

Para determinar la asociación entre las variables movilidad total y movilidad progresiva evaluadas por el método SCA ${ }^{\circledR}$, se realizó un análisis de correlación de Pearson y un análisis de regresión, de acuerdo al modelo $Y=\beta_{0}+\beta_{1} X_{1}$. Mientras para determinar la asociación entre la movilidad total medida por los métodos incluidos en estudio (Convencional y SCA ${ }^{\circledR}$ ), se efectuó un análisis de correlación de Spearman. Para todas las evaluaciones, se utilizó el programa SAS 9.2. Los resultados fueron expresados como la media \pm la desviación estándar ( $\bar{x} \pm \mathrm{DE})$.

\section{RESULTADOS Y DISCUSIÓN}

El promedio de movilidad total para el semen equino fresco fue de $83,3 \% \pm 7,6 \%$. Lozano et al. (2011) reportaron movilidades de semen equino fresco entre el 60 y el $80 \%$, mientras Candeias et al. (2012) hallaron un promedio de movilidad total de $83,5 \pm 8,1 \%$, siendo, este último, un resultado bastante cercano, al hallado en esta investigación. Para el semen post-descongelación, se obtuvo una media para la movilidad total de 59,8 $\pm 13,8 \%$, con un coeficiente de variación de 15,4\%. Se encontró un coeficiente de determinación $\left(\mathrm{R}^{2}\right)$ para el modelo ajustado de 0,73 , con lo cual, se infiere que la variabilidad en la movilidad total estuvo explicada en una alta proporción por los efectos incluidos en el modelo. Entre los efectos considerados, el equino y el método de evaluación fueron significativos $(P \leq 0,05)$, mientras la pajilla y la interacción entre la pajilla y el método, no fueron significativos $(P \leq 0,05)$. Esto muestra la importancia del equino respecto a la variabilidad en la movilidad total, así como del método, por el cual, fue evaluada la movilidad de las muestras de semen. Un total de 30 pajillas de semen equino fueron descongeladas y evaluadas mediante el sistema SCA ${ }^{\circledR}$. Los resultados para los diferentes parámetros evaluados se presentan en la tabla 1. 
Lozano et al. (2011) evaluaron, mediante un sistema CASA, la movilidad de semen equino criopreservado, encontrando valores de movilidad total y movilidad progresiva post-des- congelación, de 68,4 \pm 5,8 y $31 \pm 5,5$, respectivamente, siendo estos valores muy similares a los hallados en esta investigación. En otros estudios, mediante la evaluación se-

Tabla 1. Resultados de evaluación por un sistema SCA ${ }^{\circledR}$ de la movilidad de semen equino criopreservado.

\begin{tabular}{|c|c|c|}
\hline Parámetro & Media & Desviación estándar \\
\hline MT (\%) & 61,8 & 13,9 \\
\hline MP (\%) & 25,5 & 15,4 \\
\hline Rápidos (\%) & 16,7 & 15,1 \\
\hline Medios (\%) & 11,3 & 4,3 \\
\hline Lentos (\%) & 33,4 & 9,2 \\
\hline Inmóviles (\%) & 38,4 & 13,7 \\
\hline VCL $(\mu \mathrm{m} / \mathrm{s})$ & 55,2 & 19,9 \\
\hline VSL $(\mu \mathrm{m} / \mathrm{s})$ & 36,5 & 18,3 \\
\hline VAP $(\mu \mathrm{m} / \mathrm{s})$ & 44,6 & 21,5 \\
\hline LIN $(\%)$ & 63 & 10,7 \\
\hline STR (\%) & 80,8 & 4,9 \\
\hline ALH $(\mu \mathrm{m})$ & 2,3 & 0,4 \\
\hline BCF $(\mathrm{Hz})$ & 9,1 & 1,6 \\
\hline
\end{tabular}

MT: movilidad total; MP: movilidad progresiva; VCL: velocidad curvilínea; VSL: velocidad rectilínea; VAP: velocidad media,; LIN: índice de linealidad; STR: índice de rectitud; ALH: amplitud del desplazamiento lateral de la cabeza; BCF: frecuencia de batida.

minal con sistemas CASA y con el uso del mismo crioprotector que en esta investigación (DMF), se han registrado resultados post-descongelación de movilidad total de 52,3\% (Medeiros et al. 2002) y 90,3\% (Mesa \& Henao, 2012) y, de movilidad progresiva, de $12,6 \%$ (Medeiros et al. 2002) y $43,4 \% \pm 24,8 \%$ (Hoffmann et al. 2011). La existencia de diferencias marcadas en los resultados entre los estudios de la movilidad espermática podría ser debida al uso de diferentes protocolos de criopreservación y concentración de crioprotectores, al origen y la raza de los animales incluidos en los estudios e, incluso, a la calidad del semen sometido a la criopreservación, esta última, ligada a alta variabilidad existente, entre los reproductores y entre los eyaculados, de un mismo reproductor (Hoffmann et al. 2011).

Por otra parte, los resultados encontrados mediante el sistema SCA ${ }^{\circledR}$, para los parámetros que evalúan la velocidad de los espermatozoides (Tabla 1), fueron mayores a los reportados por Rodríguez et al. (2011), quienes hallaron promedios de VCL $(\mu \mathrm{m} / \mathrm{s})$, VAP $(\mu \mathrm{m} / \mathrm{s})$ y VSL $(\mu \mathrm{m} / \mathrm{s})$ de 40,3 $\pm 5,5,21,7$ $\pm 2,8$ y $15,6 \pm 2,6 ; 35,0 \pm 9,6,18,9 \pm 4,9$ y 13,6 \pm 4,7; $32,5 \pm 6,7,17,5 \pm 3,5$ y 12,9 $\pm 3,3$; para semen congelado con los diluyentes Caceres 1 , Gent ${ }^{\circledR}$ e INRA96 ${ }^{\circledR}$, respectiva- mente. Mientras que fueron inferiores a los reportados por Candeias et al. (2012), quienes encontraron valores de VCL $(\mu \mathrm{m} / \mathrm{s})$, VAP $(\mu \mathrm{m} / \mathrm{s})$ y VSL $(\mu \mathrm{m} / \mathrm{s})$, de $121,1 \pm 60,6,63,8 \pm$ 29,4 y $50,31 \pm 22,2$.

Son escasos los reportes de evaluación del semen de caballos de la raza criollo colombiano mediante un sistema SCA ${ }^{\circledR}$. Mesa \& Henao (2012), empleando dicho sistema y el crioprotector DMF, hallaron un valor de movilidad total post-descongelación muy superior al reportado en esta investigación (90,3\% vs. 61,8$)$; mientras el porcentaje de movilidad progresiva observado por dichos autores, fue cercano al hallado en este trabajo $(30,18 \%$ vs. 25,5$)$.

El coeficiente de correlación (Pearson) entre la movilidad total y la movilidad progresiva para la evaluación seminal en el método $\mathrm{SCA}^{\circledR}$ fue de 0,78 , lo que demuestra un alto nivel de asociación $(0,7-1,0)$, entre ambos tipos de movilidad. El coeficiente de regresión fue de 0,71 , con un coeficiente de determinación de 0,61 , para el modelo de regresión, a partir de lo cual, se infiere que se presentó un incremento de $0,71 \%$ en la movilidad total, por cada unidad de incremento en la movilidad progresiva. 
De otro lado, el coeficiente de correlación (Spearman) encontrado entre los métodos de evaluación de la movilidad total (microscópica convencional y $\mathrm{SCA}^{\circledR}$ ) fue de 0,65, demostrando una asociación media-alta $(0,4-0,69)$, entre los mismos. Adicionalmente, no se halló diferencia significativa $(P \leq 0.05)$ entre las medias de movilidad de ambos métodos (Tabla 2), con lo cual, se deduce que son igualmente apro- piados y válidos para la determinación de la movilidad total del semen equino criopreservado.

Se concluye, que el análisis de la movilidad total post-descongelación de semen equino mediante microscópica convencional es equiparable, bajo las condiciones de este estudio, al realizado mediante el sistema SCA ${ }^{\circledR}$.

Tabla 2. Movilidad total post-descongelación evaluada por los métodos convencional y SCA ${ }^{\circledR}$.

\begin{tabular}{|c|c|c|c|}
\hline Método & $\begin{array}{c}\text { Número de } \\
\text { repeticiones }\end{array}$ & Media (\%) & Desviación estándar (\%) \\
\hline Convencional & 23 & $54,3^{\mathrm{a}}$ & 10,1 \\
\hline $\mathrm{SCA}^{\circledR}$ & 30 & $61,8^{\mathrm{a}}$ & 13,9 \\
\hline
\end{tabular}

"Las letras iguales indican la no existencia de diferencia significativa $(P \leq 0.05)$.

Conflicto de intereses: Este manuscrito fue preparado y revisado con la participación de todos los autores, quienes declaramos que no existe ningún conflicto de intereses que ponga en riesgo la validez de los resultados presentados.

\section{BIBLIOGRAFÍA}

1. BUSTAMANTE, I.; PEDERZOLLI, C.; SGARAVATTI, A.; GREGORY, R.; DUTRA, C.; JOBIM, M.; MATTOS, R. 2009. Skim milk-egg yolk based semen extender compensates for non-enzymatic antioxidant activity loss during equine semen cryopreservation. Anim. Reprod. 6(2):392-399.

2. CANDEIAS, M.; ALVARENGA, M.; TEORO DO CARMO, M.; NUNES, H.; RUSSO, M.; TORRES, R.; RIOS, A.; ZANDONADI, F. 2012. Semen cryopreservation protocols of Mangalarga Marchador stallions. Rev. Bras. Zootec. 41(9):1989-1995.

3. COLENBRANDER, B.; GADELLA, B.; STOUT, T. 2003. The predictive value of semen analysis in the evaluation of the stallion fertility. Reprod. Dom. Anim. 38(4):305-311.

4. GRAHAM, J.; MOCÉ, E. 2005. Fertility evaluation of frozen/thawed semen. Theriogenology. 64:492-504.

5. HIDALGO, M.; RODRÍGUEZ, I.; DORADO, J.; SÁNZ, J.; SOLER, C. 2005. Effect of sample size and staining methods on stallion sperm morphometry by the Sperm Class Analyze. Vet. Med. Czech. 50(1):24-32.

6. HOFFMANN, N.; OLDENHOF, H.; MORANDINI, C.; ROHN, K.; SIEME, H. 2011. Optimal concentrations of cryoprotective agents for semen from stallions that are classified 'good' or 'poor' for freezing. Anim. Reprod. Sci. 125:112-118.

7. HOOGEWIJS, M.; DE VLIEGHER, S.; DE SCHAUWER, C.; GOVAERE, J.; SMITS, K.; HOFLACK, G.; DE KRUIF, A.; VAN SOOM, A. 2011. Validation and usefulness of the Sperm Quality Analyzer V equine for equine semen analysis. Theriogenology. 75:189-194.

8. JASCO, D. 1992. Evaluation of stallion semen. Vet. Clin. North. Am. Equine Pract.8(1):129-48.

9.KATILA, T. 2001. In vitro evaluation of frozen-thawed stallion semen: A review. Acta Vet. Scand. 42(2):199217.

10. KUISMA, P.; ANDERSSON, M.; KOSKINEN, E.; KATILA, T. 2006. Fertility of frozen-thawed stallion semen cannot be predicted by the currently used laboratory methods. Acta Vet. Scand. 48:14.

11. LOZANO, D.; GIL, L.; ÁLVAREZ, C. 2011. Efecto de la adición de plasma seminal en el semen equino descongelado. Sanidad Militar 67(3):284-290.

12. LOOMIS, P.R; GRAHAM, J.K 2008. Commercial semen freezing: individual male variation in cryosurvival and the response of stallion sperm to customized freezing protocols. Anim. Reprod. Sci. 105:119-128.

13. LOVE, C. 2011. Relationship between sperm motility, morphology and the fertility of stallions. Theriogenology. 76(3):547-557. 
14. MAGISTRINI, M.; VIDAMENT, M.; CLEMENET, F. 1996. Fertility prediction in stallions. Anim. Reprod. Sci. 42:181-188.

15. MEDEIROS, A.; GOMES, G.; CARMO, M.; PAPA, F.; ALVARENGA, M. 2002. Cryopreservation of stallion sperm using different amides. Theriogenology. 58:273-276.

16. MESA, A.; HENAO, G. 2012. Efecto del colesterol y la dimetilformamida sobre parámetros posdescongelación en espermatozoides de caballos criollos colombianos. Rev. MVZ Córdoba. 17(1):2908-2815.

17. MIRÓ, J.; LOBO, V.; QUINTERO-MORENO, A.; MEDRANO, A.; PEÑA, A.; RIGAU, T. 2005. Sperm motility patterns and metabolism in Catalonian donkey semen. Theriogenology 63:1706-1716.

18. MORTIMER, S. 1997. A critical review of the physiological importance and analysis of sperm movement in mammals. Hum. Reprod. Update. 3:403-439.

19. PESCH, S.; BOSTEDT, H.; FAILING, K.; BERGMANN, M. 2006. Advanced fertility diagnosis in stallion semen using transmission electron microscopy. Anim. Reprod. Sci. 91:285-298.
20. PROCTOR, J.; BOONE, W.; HIGDON, H. 2009. Comparison of the manual IVOS and SCA methods for semen analysis reporting. J. Clin. Embryol. 12(4):5-7.

21. QUINTERO, A.; MIRÓ, J.; RIGAU, A.; RODRÍGUEZ, J. 2003. Identification of sperm subpopulations with specific motility characteristics in stallion ejaculates. Theriogenology. 59:1973-1990.

22. RODRÍGUEZ, A.M.; FERRUSOLA, C.O.; GARCÍA, B.M.; MORRELL, J.M.; TAPIA, J.A.; PEÑA, J.F. 2011. Freezing stallion semen with the new Caceres extender improves post thaw sperm quality and diminishes stallion-to-stallion variability. Anim. Reprod. Sci. 27(1-2):78-83.

23. STEPHENS, D.T.; HICKMAN, R.; HOSKINS, D.D. 1988. Description, validation and performance characteristics of a new computer-automated sperm motility analysis system. Biol. Reprod. 38:577-586.

Recibido: Noviembre 21 de 2012

Aceptado: Julio 10 de 2013

Como citar:

Restrepo, G.; Ocampo, D.; Velásquez, A. 2013. Evaluación de la movilidad del semen criopreservado de caballos criollo colombiano por un sistema analizador de clase. Rev. U.D.C.A Act. \& Div. Cient. 16(2): 445-450. 